It is known that there exist specific neurodevelopmental abnormalities in the medial temporal lobe in schizophrenia. For example, pre- $\alpha$ cells in the parahippocampal gyrus show aberrant migration patterns. It is possible that glutamate abnormalities previously shown in schizoplurenia are related to neurodevelopment, and the abnormalities seen in dystrophic cells (glutamate receptors have trophic properties). We have previously shown that the expression of a particular glutamate receptor subunit (GluK1) is markedly reduced in schizophrenia (Harrison et al, Lancet 337, $\mathrm{p} 450$ ), and as a test of temporal lobe immaturity in schizophrenia, we have investigated the expression of two developmentally distinct isoforms of the glutamate receptor subunits GluRA and GluRB in the hippocampus of 17 schizophrenics and 13 age- and sex-matched control brains by the techniques of in situ hybridization and quantitative film autoradiography. Initial experiments pointed to the fact that the expression pattern of these isoforms of GluRA and GluRB was different in human hippocampus when compared to the rat, and cloning of cDNA from human frontal cortex has revealed that the two isoforms of human GluRB have virtual nucleotide sequence identity with the alternatively-spliced exons in the rat, confirming the usefulness of oligonucleotides complementary to the rat cDNAs as probes for these receptor subunits in human neuropsychiatric disorders. These results suggest that we are able to confirm these sequences as developmentally relevant in human brain tissue and facilitates a post mortem approach to understanding the developmental abnormality in schizophrenia.

\section{DOPAMINE RECEPTOR MESSENGER RNA: LOCALIZATION IN THE HUMAN HIPPOCAMPUS}

James H. Meador-Woodruff*, Jennifer Saul, Stanley J. Watson

Mental Health Research Institute and Department of Psychiatry, University of Michigan, Ann Arbor, MI 48109, USA

The dopamine receptors cluster into two families, a D 1-like family and a D2-like family. To date, five receptors have been cloned that segregate into these families: D2, D3, and D4 receptors share some "D2-like" properties, and D1 and D5 receptors are more "D1-like". While the members of each family of receptors are pharmacologically similar, the mRNAs encoding them are distributed in unique neuroanatomical profiles. All five of these mRNAs, however, appear to be distributed within the hippocampus of the rat. Because of this apparently common site of distribution, as well as various lines of evidence suggesting the involvement of the hippocampus and associated cortex in the pathogenesis of schizophrenia, we have defined the normal neurochemical anatomy of the five dopamine receptor mRNAs in the human hippocampus using in situ hybridization. All 5 mRNAs were found to exist within the hippocampus as well as in associated cortex, but each was uniquely distributed. This study begins to establish a normative database for our subsequent planned studies in schizophrenia, as well as allowing the development of a clearer understanding of the dopaminergic innervation of these temporal lobe structures.

Supported by MH42251 and MH00818.

\section{DIFFERENTIAL INDUCTION OF NEUROTENSIN AND C-fos GENE EXPRESSION BY TYPICAL VERSUS ATYPICAL ANTIPSYCHOTICS IN STRIATAL REGIONS OF THE RAT BRAIN}

\author{
Kalpana M. Merchant*, Daniel M. Dorsa \\ Department of Psychiatry and Behavioral Sciences (RP 10), \\ University of Washington School of Medicine, Seattle, WA \\ 98195, USA
}

Present studies investigated the effects of a variety of antipsychotic drugs on the expression of the gene encoding the purported endogenous antipsychotic-like peptide, neurotensin (NT), in striatal regions of the rat brain. Several clinically efficacious antipsychotic drugs selectively increased the expression of NT/N mRNA in the shell of the nucleus accumbens, a region thought to be involved in antipsychotic effects. On the other hand, only typical antipsychotics that cause a high incidence of acute EPS increased the expression of NT/N mRNA in the dorsolateral striatum, an extrapyramidal region primarily involved in motor control. In addition, neuroleptic-induced increases in NT/N mRNA expression in the dorsolateral striatum, but not the accumbal shell, were accompanied by a rapid and transient activation of $c$-fos mRNA. The site specific differential activation of NT gene expression raise the possibility that (a) NT neurons may contribute to the therapeutic effects (neurons in the accumbal shell) or motor side effects (neurons in the dorsolateral striatum) of the antipsychotic drugs and (b) alterations in NT/N and $c$-fos mRNA induced by antipsychotic drugs may be used as a preclinical screening assay to predict acute EPS liability of new psychoactive drugs.

\section{ACUTE EFFECTS OF NEUROLEPTICS ON UNMEDICATED SCHIZOPHRENICS AND CONTROLS}

Alexander L. Miller*, James W. Maas, Salvador Contreras, Ermias Seleshi, Janet E. True, Charles Bowden, Joseph Castiglioni

Department of Psychiatry, University of Texas Health Science Center at San Antonio and Audie Murphy VA Medical Center, San Antonio, TX 78284, USA

Many studies have suggested that the psychosis of schizophrenia is associated with a hyperdopaminergic state. Other reports suggest increased CNS output of norepinephrine in the psychosis of schizophrenia. If schizophrenics and controls differ from one another in their CNS dopamine metabolism, acute administration 